Pacific

Journal of

Mathematics

EXTENSIONS OF TORI IN SL(2)

JefFrey AdAms

Volume $200 \quad$ No. 2

October 2001 


\title{
EXTENSIONS OF TORI IN SL(2)
}

\author{
JEFFREY ADAMS
}

Let $S \widetilde{L(2, \mathbb{F})}$ be the metaplectic two-fold cover of $S L(2, \mathbb{F})$, the special linear group in two variables over a local field $\mathbb{F}$ of characteristic 0 . The inverse image $\widetilde{T}$ of a maximal torus $T$ in $S \widetilde{L(2, \mathbb{F})}$ is an abelian extension of $T$ by \pm 1 . We consider the question of whether this extension is trivial. More generally we find the minimal subgroup $A$ of the circle for which the extension is split when considered with coefficients in $A$. We see that $|A|=2,4$ or 8 in the p-adic case. We also find an explicit splitting function for the cocycle.

\section{Introduction}

Let $\widehat{S L(2, \mathbb{F})}$ be the metaplectic two-fold cover of $S L(2, \mathbb{F})$, the special linear group in two variables over a local field $\mathbb{F}$ of characteristic 0 . The inverse image $\widetilde{T}$ of a maximal torus $T$ in $\widehat{S L(2, \mathbb{F})}$ is an abelian extension of $T$ by \pm 1 . We consider the question of whether this extension is trivial. We exclude the case $\mathbb{F}=\mathbb{C}$, which is trivial.

More generally suppose $A$ is a subgroup of the circle $\mathbb{T}$ containing \pm 1 . The inclusion of \pm 1 in $A$ induces a map on cohomology, and defines an extension

$$
1 \rightarrow A \rightarrow T_{A} \rightarrow T \rightarrow 1 .
$$

We say $A$ is a splitting group for $\widetilde{T}$ if the extension $T_{A} \rightarrow T$ splits. It is well-known that $\mathbb{T}$ is a splitting group. We say a splitting group $A$ is a minimal splitting group if no proper subgroup of $A$ is a splitting group. It is easy to see the order of a minimal splitting group is a power of 2 , and hence unique, if it is finite.

Let $(,)_{\mathbb{F}}$ be the Hilbert symbol of $\mathbb{F}$, and let $\mu_{n}$ be the $n^{\text {th }}$ roots of unity in $\mathbb{C}$.

Theorem 1. The minimal splitting group $A_{\min }$ for $T$ is given by:

(a) Suppose $T \simeq \mathbb{F}^{*}$. Then

$$
A_{\text {min }}= \begin{cases}\mu_{2} & (-1,-1)_{\mathbb{F}}=1 \\ \mu_{4} & (-1,-1)_{\mathbb{F}}=-1 .\end{cases}
$$


(b) Suppose $T \simeq \mathbb{E}^{1}$ for $\mathbb{E}$ a quadratic extension of $\mathbb{F}$. Then

$$
A_{\min }= \begin{cases}\mu_{2} & (-1,-1)_{\mathbb{F}}=1 \\ \mu_{4} & (-1,-1)_{\mathbb{F}}=-1, \mathbb{F} \text { non-archimedean, }-1 \notin \mathbb{E}^{* 2} \\ \mu_{8} & (-1,-1)_{\mathbb{F}}=-1, \mathbb{F} \text { non-archimedean, }-1 \in \mathbb{E}^{* 2} \\ \mathbb{T} & \mathbb{F}=\mathbb{R} .\end{cases}
$$

Remark 2. It is well-known that $(-1,-1)_{\mathbb{F}}=1$ unless $\mathbb{F}=\mathbb{R}, \mathbb{Q}_{2}$, or an extension of $\mathbb{Q}_{2}$ of odd degree.

Theorem 1 is proved in Sections 3, 4 and 5. Here is an alternative realization of $\widetilde{T}$. A character of $\widetilde{T}$ is said to be genuine if it does not factor to $T$.

Theorem 3. Let $\tau(z)=z^{2}\left(z \in \mathbb{C}^{*}\right)$. Let $\widetilde{\alpha}$ be a genuine character of $\widetilde{T}$. Then $\widetilde{\alpha}^{2}$ factors to a character $\alpha$ of $T$, and $\widetilde{T}$ is isomorphic to the pullback of $\tau$ via $\alpha$. In other words $\widetilde{T}$ is isomorphic to the $\sqrt{\alpha}$-extension of $G$.

From this we obtain an interpretation of the minimal splitting group of Theorem 1. Let $n(\widetilde{T})$ be the minimal order of a genuine character of $\widetilde{T}$. Set $\mu_{\infty}=\mathbb{T}$.

Corollary 4. The minimal splitting group for $\widetilde{T}$ is $\mu_{n(\widetilde{T})}$.

For the proofs of Theorem 3 and Corollary 4 see Lemma 1.4.

We also give an explicit splitting of this extension, i.e., a function $\zeta: T \rightarrow$ $A_{\text {min }}$ whose coboundary is the cocycle defining $\widetilde{T}$ (see $\S 3$ and Theorem 5.7).

These questions arise from the theory of the oscillator representation and dual pairs. The splitting plays a role in this context, for example see [11]. The case of $\mathbb{F}^{*}$ is well-known ([4], p. 42, attributed to J. Klose), as is the existence of a $\mathbb{T}$-splitting in general [2]. General results about the splitting of the metaplectic cover over subgroups are due to Kudla [7], and a splitting of the extension of an elliptic torus is found in [7], Proposition 4.8 (in the non-archimedean case it is easy to see this can be taken to be a $\mu_{8}$-splitting). This paper grew out of an effort to simplify Kudla's formula. In the case of a p-adic field of odd residual characteristic a formula for a $\mu_{2}$-splitting in some cases may be deduced from [6], cf. ([4], p. 43).

Many of the arguments, especially those of Section 1 apply to other abelian extensions of abelian groups, for example a maximal torus in the two-fold cover of $S p(2 n, \mathbb{F})$. If $\widetilde{G}$ is a non-linear $n$-fold cover of the $\mathbb{F}$ points of an algebraic group $G$, then the inverse image $\widetilde{T}$ of a maximal torus in $G$ is typically not abelian. However similar arguments apply to the center of $\widetilde{T}$.

Throughout $\mathbb{F}$ denotes a local field of characteristic zero, and $(x, y)_{\mathbb{F}} \in \mu_{2}$ is the Hilbert symbol. For $x \in \mathbb{F}^{*}$ and $\psi_{\mathbb{F}}$ a non-trivial additive character of 
$\mathbb{F}, \gamma_{\mathbb{F}}\left(x, \psi_{\mathbb{F}}\right) \in \mu_{4}$ is the Weil index. We use basic properties of the Hilbert symbol and the Weil index without further comment, see ([10], Appendix) for details. We make repeated use of the identities

$$
\begin{aligned}
\gamma_{\mathbb{F}}\left(x, \psi_{\mathbb{F}}\right) \gamma_{\mathbb{F}}\left(y, \psi_{\mathbb{F}}\right) & =(x, y)_{\mathbb{F}} \gamma_{\mathbb{F}}\left(x y, \psi_{\mathbb{F}}\right) \\
\gamma_{\mathbb{F}}\left(x, \psi_{\mathbb{F}}\right)^{2} & =(-1, x)_{\mathbb{F}} .
\end{aligned}
$$

If $\mathbb{E}$ is a quadratic extension of $\mathbb{F}$ then

$$
\begin{aligned}
(x, z)_{\mathbb{E}} & =(x, N z)_{\mathbb{F}} \quad\left(x \in \mathbb{F}^{*}, z \in \mathbb{E}^{*}\right) \\
(x, y)_{\mathbb{E}} & =1 \quad\left(x, y \in \mathbb{F}^{*}\right) \\
\gamma_{\mathbb{E}}\left(x, \psi_{\mathbb{E}}\right) \gamma_{\mathbb{E}}\left(y, \psi_{\mathbb{E}}\right) & =\gamma_{\mathbb{E}}\left(x y, \psi_{\mathbb{E}}\right) \quad\left(x, y \in \mathbb{F}^{*}\right) .
\end{aligned}
$$

I would like to thank Steve Kudla and Jonathan Rosenberg for many useful discussions, and Jim Schafer for assistance with the arguments in Section 1.

\section{Abstract Groups.}

In this section we ignore the topology on $T$ and consider it as an abstract group. We recall some standard facts from group cohomology and establish some notation. For example see [1].

Suppose $G$ is a group, $A$ is an abelian group, and $G$ acts trivially on $A$. The equivalence classes of central extensions of $G$ by $A$ are parametrized by the group cohomology $H^{2}(G, A)$. Given an extension $p: H \rightarrow G$ let $s: G \rightarrow H$ be a section, i.e., $p \circ s=1$. The cohomology class of the extension is represented by the 2-cocycle $c_{s}(g, h)=s(g h) s(h)^{-1} s(g)^{-1}$. When there is no danger of confusion we do not distinguish between $c_{s}$ and its image $\bar{c}_{s}$ in $H^{2}(G, A)$. Any other such splitting $s^{\prime}$ is given by $s^{\prime}(g)=s(g) \zeta(g)$ for some map $\zeta: G \rightarrow A$, and then $c_{s^{\prime}}(g, h)=c(g, h) \zeta(g h) \zeta(h)^{-1} \zeta(g)^{-1}$. Thus $c_{s^{\prime}}=c_{s} d \zeta$, and $\bar{c}_{s}=\bar{c}_{s^{\prime}}$.

Conversely given a cocycle $c$ we define $H$ to be equal to $G \times A$ as a set, with multiplication $(g, a)\left(g^{\prime}, a^{\prime}\right)=\left(g g, a a^{\prime} c\left(g, g^{\prime}\right)\right)$. The cocyle $c$ is trivial in cohomology if and only if

$$
c(g, h)=\zeta(g) \zeta(h) \zeta(g h)^{-1}
$$

for some $\zeta$, i.e., $d \zeta=c$. We say $\zeta$ is a splitting of the cocycle. Equivalently the splitting map $s(g)=\left(g, \zeta^{-1}(g)\right)$ is a homomorphism. Any other splitting is then of the form $\zeta^{\prime}=\zeta \alpha$ with $\alpha: G \rightarrow A$ a homomorphism.

Suppose $A=\mu_{2}$, with cocycle $c$, and $A \subset \mu_{a b}$ with $b$ odd. If $\zeta: G \rightarrow$ $\mu_{a b}$ is a splitting of $c$, then $\zeta^{b}$ is a $\mu_{a}$ splitting. Therefore we will restrict consideration to $\mu_{n}$ with $n$ a power of 2 .

Now suppose $G$ is abelian. The universal coefficient theorem for group cohomology gives an exact sequence:

$$
1 \rightarrow \operatorname{Ext}(G, A) \rightarrow H^{2}(G, A) \stackrel{\phi}{\rightarrow} \operatorname{Hom}\left(\Lambda^{2} G, A\right) \rightarrow 1 .
$$


Here $G$ and $A$ are considered as $\mathbb{Z}$-modules, Hom $=$ Hom $_{\mathbb{Z}}$, Ext $=$ Ext $_{\mathbb{Z}}$, and $\operatorname{Hom}\left(\Lambda^{2} G, A\right)$ consists of alternating, bilinear maps $G \times G \rightarrow A$.

If $c$ is a 2-cocycle, representing the class $\bar{c} \in H^{2}(G, A)$, then $\phi(\bar{c})(g, h)=$ $c(g, h) c(h, g)^{-1}$. In terms of the group, suppose $p: H \rightarrow G$ is the corresponding extension. For $g, h \in G$ and any section $s$ let $\{g, h\}$ be the commutator $s(g) s(h) s(g)^{-1} s(h)^{-1}$. This is contained in $A$, is independent of the choice of $s$, and $\phi(\bar{c})(g, h)=\{g, h\}$. In particular $\phi(\bar{c})=1$ if and only if $H$ is abelian, so $\operatorname{Ext}(G, A) \subset H^{2}(G, A)$ parametrizes the abelian extensions of $G$ by $A$.

Let $G^{n}=\left\{g^{n} \mid g \in G\right\}$ and ${ }_{n} G=\left\{g \in G \mid g^{n}=1\right\}$. The next result is presumably well-known to the experts.

Lemma 1.1. For any positive integer $n$, inclusion $\iota:{ }_{n} G \hookrightarrow G$ induces an isomorphism:

$$
\operatorname{Ext}\left(G, \mu_{n}\right) \simeq \operatorname{Ext}\left({ }_{n} G, \mu_{n}\right) .
$$

Proof. Consider the maps

$$
G \stackrel{\alpha}{\rightarrow} G^{n} \stackrel{\beta}{\rightarrow} G
$$

where $\alpha(g)=g^{n}$ and $\beta$ is inclusion. The induced map $\alpha^{*} \beta^{*}: \operatorname{Ext}\left(G, \mu_{n}\right) \rightarrow$ $\operatorname{Ext}\left(G, \mu_{n}\right)$ is induced by the $n^{\text {th }}$ power map $g \rightarrow g^{n}$ on $G$. This is the same map as that induced by the $n^{\text {th }}$ power map on $\mu_{n}$, and therefore $\alpha^{*} \beta^{*}=0$.

Now the long exact cohomology sequence corresponding to $0 \rightarrow G^{n} \stackrel{\beta}{\rightarrow}$ $G \rightarrow G / G^{n} \rightarrow 0$ has final two terms $\operatorname{Ext}(G, A) \stackrel{\beta^{*}}{\rightarrow} \operatorname{Ext}\left(G^{n}, A\right) \rightarrow 0$. Therefore $\beta^{*}$ is surjective, which implies $\alpha^{*}=0$. On the other hand the short exact sequence

$$
0 \rightarrow{ }_{n} G \stackrel{\iota}{\rightarrow} G \stackrel{\alpha}{\rightarrow} G^{n} \rightarrow 0
$$

gives rise to the long exact sequence

$$
\begin{array}{r}
0 \rightarrow \operatorname{Hom}\left(G^{n}, A\right) \rightarrow \operatorname{Hom}(G, A) \rightarrow \operatorname{Hom}\left({ }_{n} G, A\right) \rightarrow \\
\operatorname{Ext}\left(G^{n}, A\right) \stackrel{\alpha^{*}}{\rightarrow} \operatorname{Ext}(G, A) \stackrel{\iota^{*}}{\rightarrow} \operatorname{Ext}\left({ }_{n} G, A\right) \rightarrow 0 .
\end{array}
$$

Since $\alpha^{*}=0, \iota^{*}$ is an isomorphism.

Remark 1.2. In our setting ${ }_{2} T= \pm 1$. For the $\mu_{2}$ extension $\widetilde{T}$ to split it is necessary that it splits over \pm 1 . Perhaps surprisingly the converse holds as well by the Lemma.

For later use we note an explicit formula for a splitting of $\alpha^{*} \beta^{*} c$. We drop the assumption that $H$ is abelian, so let $p: H \rightarrow G$ be an extension, with section $s$ and corresponding cocycle $c$.

Lemma 1.3. Let

$$
\begin{aligned}
\tau(g) & =s\left(g^{n}\right) s(g)^{-n} \\
& =c(g, g)^{-1} c\left(g, g^{2}\right)^{-1} \ldots c\left(g, g^{n-1}\right)^{-1} \in A .
\end{aligned}
$$


Then

$$
c\left(g^{n}, h^{n}\right)=\tau(g) \tau(h) \tau(g h)^{-1}\{g, h\}^{n(n-1) / 2} .
$$

If $H$ is abelian then $d \tau=\alpha^{*} \beta^{*} c$.

Note that $\{g, h\}^{n(n-1) / 2}= \pm 1$, and is identically 1 if $n$ is odd. Compare ([3], p. 130) and ([5], §4).

Proof. This follows from the identity

$$
[s(g) s(h)]^{n}=s(g)^{n} s(h)^{n}\{h, g\}^{n(n-1) / 2} .
$$

Using $s(g) s(h)=s(g h) c(g, h)$ and $s(g)^{n}=s\left(g^{n}\right) \tau(g)^{-1}$, the left hand side is equal to

$$
s(g h)^{n}=s\left(g^{n} h^{n}\right) \tau(g h)^{-1} .
$$

The right hand side is

$$
\begin{aligned}
& s\left(g^{n}\right) s\left(h^{n}\right) \tau(g)^{-1} \tau(h)^{-1}\{h, g\}^{n(n-1) / 2} \\
& =s\left(g^{n} h^{n}\right) c\left(g^{n}, h^{n}\right) \tau(g)^{-1} \tau(h)^{-1}\{h, g\}^{n(n-1) / 2}
\end{aligned}
$$

and the first assertion follows. Since $\alpha^{*} \beta^{*} c(g, h)=c\left(g^{n}, h^{n}\right)$, the second assertion is equivalent to

$$
c\left(g^{n}, h^{n}\right)=\tau(g) \tau(h) \tau(g h)^{-1}
$$

which is (8) for $H$ abelian.

For $H$ an abelian extension (9) implies $\tau$ is a character when restricted to ${ }_{n} G$. In terms of the exact sequence (7), $c \in \operatorname{Ext}(G, A), \beta^{*} c \in \operatorname{Ext}\left(G^{n}, A\right)$, $\alpha^{*} \beta^{*} c=0$, and $\beta^{*} c$ is the image of $\tau \in \operatorname{Hom}\left({ }_{n} G, A\right)$. Thus $\beta^{*} c=0$ if $\tau$ extends to an element of $\operatorname{Hom}(G, A)$.

More generally, we try to find a splitting subgroup $A$ of $\beta^{*} c$, i.e., $c$ restricted to $G^{n}$, together with an explicit formula. Note that (9) does not necessarily define such a splitting since the function $g^{n} \rightarrow \tau(g)$ is not necessarily well-defined. Let $\alpha$ be a character of $G$ whose restriction to ${ }_{n} G$ is equal to $\tau^{-1}$. Then $\zeta_{\alpha}\left(g^{n}\right):=\tau(g) \alpha(g)$ is well-defined, and $d \zeta_{\alpha}=c$. The minimal splitting subgroup for $\beta^{*} c$ is thus the minimal subgroup $A$ of $\mathbb{T}$, containing $\mu_{n}$, such that $\tau$ restricted to ${ }_{n} G$ can be extended to a character of $G$ with values in $A$.

\section{Characters and the $\sqrt{\alpha}$ extension.}

For the remainder of this section let $p: \widetilde{G} \rightarrow G$ be a $\mu_{2}$ extension of a group $G$. We do not assume that $G$ or $\widetilde{G}$ is abelian. If $\alpha$ is a character of $G$, and $\tau(z)=z^{2}\left(z \in \mathbb{C}^{*}\right)$ then the pullback of $\tau$ via $\alpha$ is a $\mu_{2}$ extension of $G$, and may be realized as the subgroup of $G \times \mathbb{C}^{*}$ given by $\{(g, z) \mid \alpha(g)=\tau(z)\}$. Projection on the second factor is a genuine character $\widetilde{\alpha}$ of $\widetilde{G}$ satisfying $\widetilde{\alpha}^{2}=\alpha \circ p$. This is sometimes denoted the $\sqrt{\alpha}$-extension of $G$. It may or may not be the trivial extension. 
We see that $\widetilde{G}$ has a $\mathbb{T}$-splitting if and only if there is a genuine character of $\widetilde{G}$. More precisely:

Lemma 1.4. Suppose there is a genuine character $\widetilde{\alpha}$ of $\widetilde{G}$. Then $\widetilde{\alpha}^{2}$ factors to a character $\alpha$ of $G$, and $\widetilde{G}$ is isomorphic to the $\sqrt{\alpha}$ extension of $G$. If Image $(\widetilde{\alpha}) \subset A \subset \mathbb{T}$ then $G_{A} \simeq G \times A$. The minimal splitting group for $G$ is $\mu_{n(\widetilde{G})}$ where $n(\widetilde{G})$ is the minimal order of a genuine character of $\widetilde{G}$.

Conversely if $\widetilde{G}_{A} \simeq G \times A$ then there is a genuine character of $\widetilde{G}$ with values in $A$.

Note that there exists a genuine character $\widetilde{\alpha}$ of $\widetilde{G}$ if and only if $z \notin[\widetilde{G}, \widetilde{G}]$ where $z$ is the non-trivial element in the inverse image of 1 . In particular this holds if $\widetilde{G}$ is abelian, which proves the existence of a $\mathbb{T}$-splitting (cf. $[2])$.

Proof. The map $\phi: g \rightarrow(p(g), \widetilde{\alpha}(g)) \subset G \times \mathbb{T}$ is an isomorphism of $\widetilde{G}$ with the pullback of $\tau$ via $\alpha$. This is a subgroup of $G \times A$, and $\phi$ extends to an isomorphism of $\widetilde{G}_{A}$ with $G \times A$. The final two assertions are immediate.

Remark 1.5. In the setting of the Lemma, suppose $\zeta$ is a $\mathbb{T}$-splitting of the cocycle defining $\widetilde{G}$ (with respect to a section $s$ ). Then $\alpha:=\zeta^{2}$ is a character of $G$, and $\widetilde{G}$ is isomorphic to the $\sqrt{\alpha}$ cover of $G$.

Theorem 3 and Corollary 4 are immediate consequences of the Lemma. Theorem 1 also follows from the Lemma, from a computation of $n=n(\widetilde{T}): n$ is the minimal power of 2 such that $z \notin \widetilde{T}^{n}$. We follow a different approach, by giving explicit formulas for the minimal splitting $\zeta$ in Sections 3-5.

\section{Moore cohomology and $\widetilde{S L(2, \mathbb{F})}$.}

Now suppose $G$ and $A$ are locally compact topological groups, $A$ is abelian, and $G$ acts continuously on $A$. In our applications $A$ will either be $\mu_{n}$ or $\mathbb{T}$, with trivial $G$ action. C. Moore has defined cohomology groups $H_{\text {top }}^{n}(G, A)$ using measurable cochains [8]. In the case of a totally disconnected group it is equivalent to use continuous cochains. Viewing $G$ and $A$ as abstract groups, there is a natural homomorphism $H_{\text {top }}^{2}(G, A) \rightarrow H^{2}(G, A)$. In general it is neither surjective nor injective.

Now let $G=G L(2, \mathbb{F})$. We recall the definition of the standard cocycle on $G[6]$, cf. ([4], p. 41). For $g=\left(\begin{array}{ll}a & b \\ c & d\end{array}\right)$ let $x(g)=c($ resp. $d)$ if $c \neq 0$ (resp. $c=0)$. Then

$$
c(g, h)=(x(g) x(g h), x(h) x(g h))_{\mathbb{F}}(\operatorname{det}(g), x(g) x(g h))_{\mathbb{F}} .
$$


This defines a $\mu_{2}$-extension $\widetilde{G L(2, \mathbb{F})}$ of $G L(2, \mathbb{F})$, with distinguished section $s$. We write $\widehat{G L(2, \mathbb{F})}$ in cocycle notation as usual. The restriction to $S L(2, \mathbb{F})$ is isomorphic to $\widehat{S L(2, \mathbb{F})}$.

Up to conjugation $G L(2, \mathbb{F})$ contains one hyperbolic torus isomorphic to $\mathbb{F}^{*} \times \mathbb{F}^{*}$, and for each quadratic extension $\mathbb{E}$ of $\mathbb{F}$ one elliptic torus isomorphic to $\mathbb{E}^{*}$. The commutator of two elements $z, w$ of $\mathbb{E}^{*}$ is given by $([\mathbf{3}]$, p. 128)

$$
\begin{aligned}
\{z, w\} & =(z, \bar{w})_{\mathbb{E}} \\
& =(z, w)_{\mathbb{E}}(N z, N w)_{\mathbb{F}} .
\end{aligned}
$$

Here and elsewhere we suppress the map $\iota: \mathbb{E}^{*} \hookrightarrow G L(2, \mathbb{F})$ from the notation, and write $\{z, w\}:=\{\iota(z), \iota(w)\}$. The commutator is trivial when restricted to $\mathbb{E}^{1}=\mathbb{E}^{*} \cap S L(2, \mathbb{F})$.

\section{The hyperbolic torus.}

Let $\iota: \mathbb{F}^{*} \hookrightarrow S L(2, \mathbb{F})$, so $T=\iota\left(\mathbb{F}^{*}\right)$ is a hyperbolic torus. After conjugation we may assume $\iota(x)=\operatorname{diag}\left(x, x^{-1}\right)$. We drop $\iota$ from the notation and identify $x$ with $\iota(x)$. We prove Theorem 1 in this case, together with a formula for the minimal splitting. While these results are well-known they are not easy to find in the literature, and the calculation illustrates some of the ideas in the next section.

The restriction of $\widehat{S L(2, \mathbb{F})}$ to $T$ defines a $\mu_{2}$ extension $\widetilde{T}$ of $T$ with cocycle

$$
c(x, y)=(x, y)_{\mathbb{F}} .
$$

In particular $(-I, \epsilon)^{2}=\left(I,(-1,-1)_{\mathbb{F}}\right)$, so the restriction of the extension to $\pm I$ is isomorphic to $\mathbb{Z} / 2 \mathbb{Z} \times \mathbb{Z} / 2 \mathbb{Z}$ if $(-1,-1)_{\mathbb{F}}=1$, or $\mathbb{Z} / 4 \mathbb{Z}$ if $(-1,-1)_{\mathbb{F}}=$ -1 . By Lemma 1.1 the minimal splitting group for $\widetilde{T}$, considered as an abstract group, is $\mu_{2}$ (resp. $\left.\mu_{4}\right)$ if $(-1,-1)_{\mathbb{F}}=1$ (resp. -1$)$. It remains to show this splitting is measurable. We do this by computing it explicitly.

Fix $\psi$ and write $\gamma(x)=\gamma_{\mathbb{F}}(x, \psi)$. The key point is that properties (1) and (2) of the Weil index shows that $d \gamma=c$ and $\gamma^{4}=1$, so $\gamma$ is a measurable $\mu_{4}$ splitting of $c$. This completes the proof in case $(-1,-1)_{\mathbb{F}}=-1$, so assume $(-1,-1)_{\mathbb{F}}=1$. Let $\alpha$ be a character of $\mathbb{F}^{*}$ satisfying $\alpha\left(x^{2}\right)=(-1, x)_{\mathbb{F}}$. To see that such a character exists, define $\alpha$ restricted to $\mathbb{F}^{* 2}$ by this formula; it is well-defined since $\alpha\left((-x)^{2}\right)=(-1,-x)_{\mathbb{F}}=(-1,-1)_{\mathbb{F}} \alpha\left(x^{2}\right)=\alpha\left(x^{2}\right)$. Extend arbitrarily from $\mathbb{F}^{* 2}$ to $\mathbb{F}^{*}$. By $(2) \alpha(x)^{2} \gamma(x)^{2}=(-1, x)_{\mathbb{F}}^{2}=1$. Let $\zeta_{\alpha}=\gamma \alpha$, i.e.,

$$
\zeta_{\alpha}(x)=\gamma\left(x, \psi_{\mathbb{F}}\right) \alpha(x) .
$$

Then $d \zeta_{\alpha}=d \gamma=c$ and $\zeta_{\alpha}$ is a $\mu_{2}$-splitting of $c$.

Choose representatives $a_{1}, a_{2}, \ldots, a_{m} \in \mathbb{F}^{*}$ of generators of $\mathbb{F}^{*} / \mathbb{F}^{* 2} \simeq$ $(\mathbb{Z} / 2 \mathbb{Z})^{m}$. (By $[4]$, Lemma $0.3 .2,2^{m}=4 /|2|_{\mathbb{F}}$.) Given any choice of signs 
$\epsilon_{i}$ we may choose $\alpha$ so that $\zeta\left(a_{i}\right)=\epsilon_{i}$, and $\alpha\left(x^{2}\right)=(-1, x)_{\mathbb{F}}$ for all $x \in \mathbb{F}$. Then $\zeta$ extends uniquely to a splitting.

For example, if $-1 \in \mathbb{F}^{* 2}$ then we may take $\alpha=1$ and $\zeta(x)=\gamma_{\mathbb{F}}\left(x, \psi_{\mathbb{F}}\right)=$ \pm 1 . On the other hand, suppose $-1 \notin \mathbb{F}^{* 2}$ and the residual characteristic of $\mathbb{F}$ is odd. We may take representatives $\pm 1, \pm \varpi$ for $\mathbb{F}^{*} / \mathbb{F}^{* 2}$ ( $\varpi$ is a uniformizing parameter) and then choose $\zeta$ satisfying:

$$
\begin{aligned}
\zeta\left( \pm x^{2}\right) & =(-1, x)_{\mathbb{F}} \\
\zeta\left( \pm \varpi x^{2}\right) & = \pm(-1, x)_{\mathbb{F}} .
\end{aligned}
$$

\section{Elliptic tori.}

Let $T$ be an elliptic torus of $S L(2, \mathbb{F})$ as in $\S 2$. Thus $\mathbb{E}$ is a quadratic extension of $\mathbb{F}, \iota: \mathbb{E}^{1} \hookrightarrow S L(2, \mathbb{F})$ is an embedding, and $T=\iota\left(\mathbb{E}^{1}\right)$. As in $\S 3$ we fix $\iota$ and drop it from the notation.

As in the case of the hyperbolic torus, $(-I, \epsilon)$ has order 2 or 4 depending on whether $(-1,-1)_{\mathbb{F}}=+1$ or -1 . By Lemma 1.1 this proves $\mu_{2}$ is a splitting group if and only if $(-1,-1)_{\mathbb{F}}=1$, so assume $(-1,-1)_{\mathbb{F}}=-1$.

Let $\mathbb{F}=\mathbb{R}, \mathbb{E}=\mathbb{C}$. Since $T=T^{2}$, it is enough to find a splitting of $\beta^{*} c$ as in $\S 2$. Choose a character $\alpha$ of $\mathbb{E}^{1}$ such that $\alpha(-1)=-1$, i.e., $\alpha(z)=z^{n}$ for $n$ odd. It is easy to see $c(-z,-z)=-c(z, z)$, and the discussion in $\S 2$ shows that

$$
\zeta_{\alpha}\left(z^{2}\right):=c(z, z) \alpha(z)
$$

is a well-defined (measurable) $\mathbb{T}$-splitting. Since $\zeta_{\alpha}$ is surjective onto $\mathbb{T}$ for any $\alpha$, this shows that there is no $A$-splitting for any proper subgroup $A$ of $\mathbb{T}$.

We now assume $\mathbb{F}$ is non-archimedean. Since $(-1,-1)_{\mathbb{F}}=-1, \mathbb{F}$ is an extension of $\mathbb{Q}_{2}$ of odd degree, and $-1 \notin \mathbb{F}^{* 2}$. If $-1 \notin \mathbb{E}^{* 2}$ then ${ }_{4} T=\{ \pm 1\}$. Since $(-I, \zeta)^{4}=\left(I, \pm \zeta^{2}\right)^{2}=I$ for all $\zeta \in \mu_{4}$, the $\mu_{4}$-extension splits over ${ }_{4} T$.

Suppose $-1=\delta^{2}\left(\delta \in \mathbb{E}^{*}\right)$. We claim ${ }_{4} T={ }_{8} T=\{ \pm 1, \pm \delta\}$. It is enough to show $\mathbb{E}^{*}$ does not contain a primitive eighth root of unity, or equivalently $\delta \notin \mathbb{E}^{* 2}$. Since $\mathbb{F}$ is an extension of $\mathbb{Q}_{2}$ of odd degree, $2 \notin \mathbb{F}^{* 2}$. But then $(a+b \delta)^{2}=\delta$ implies $a^{2}= \pm \frac{1}{2}$, which is a contradiction.

For any $\zeta \in \mu_{8}$ we compute $(\delta, \zeta)^{8}=\left(-I, \pm \zeta^{2}\right)^{4}=\left(I,-\zeta^{4}\right)^{2}=I$, which implies the extension splits over ${ }_{8} T$.

Therefore $\mu_{4}$ (resp. $\mu_{8}$ ) is a minimal splitting group for $T$ if $\mathbb{F}$ is nonarchimedean, $(-1,-1)_{\mathbb{F}}=-1$, and $-1 \notin \mathbb{E}^{* 2}$ (resp. $-1 \in \mathbb{E}^{* 2}$ ). It remains to show these splittings can be chosen to be measurable. In the next section we give explicit such splittings.

Remark 4.1. We have shown the cohomology class $\bar{c} \in H_{\text {top }}^{2}(G, A)$ has image 0 in $H^{2}(G, A)$ in the given cases. An argument due to Jonathan 
Rosenberg shows that the map $H_{\mathrm{top}}^{2}(G, A) \rightarrow H^{2}(G, A)$ is injective in this situation. Since we are interested in explicit formulas for the splittings in any case, we do not pursue this approach. For $G$ perfect (not at all the case here!) the injectivity of $\phi$ is known ([9], Theorem 2.3).

\section{Explicit splittings for elliptic tori.}

We continue with the notation of the previous section. The embedding $\iota: \mathbb{E}^{1} \hookrightarrow S L(2, \mathbb{F})$ extends to an embedding $\iota: \mathbb{E}^{*} \hookrightarrow G L(2, \mathbb{F})$. We will make use of the non-abelian $\mu_{2}$ extension of $\mathbb{E}^{*}$ obtained by restricting the extension $\widehat{G L(2, \mathbb{F})}$ of $G L(2, \mathbb{F})$.

We proceed as follows. Since $\{z, w\}=(z, \bar{w})_{\mathbb{E}}$, the commutator is trivial on $\mathbb{E}^{* 2}$. By the method of $\S 1$ we find a splitting of this extension. As in $\S 3$ we also find a splitting of the extension of $\mathbb{F}^{*} \subset \mathbb{E}^{*}$. The extension of $\mathbb{E}^{* 2} \mathbb{F}^{*}$ is abelian, and by an explicit version of the Mayer-Vietoris sequence we obtain a splitting of this extension. Finally $\mathbb{E}^{1}$ is contained in $\mathbb{E}^{* 2} \mathbb{F}^{*}$, and we restrict to obtain a splitting of the extension of $\mathbb{E}^{1}$.

Fix non-trivial additive characters $\psi_{\mathbb{F}}$ of $\mathbb{F}$ and $\psi_{\mathbb{E}}$ of $\mathbb{E}$. Recall (5) the restriction of $\gamma_{\mathbb{E}}\left(\cdot, \psi_{\mathbb{E}}\right)$ to $\mathbb{F}^{*}$ is a quadratic character.

Lemma 5.1. Suppose $\lambda$ (respectively $\mu$ ) is a splitting of the cocycle restricted to $\mathbb{E}^{* 2}$ (respectively $\left.\mathbb{F}^{*}\right)$. Assume $\lambda(x)=\mu(x)$ for $x \in \mathbb{E}^{* 2} \cap \mathbb{F}^{*}$. For $z \in \mathbb{E}^{*}, x \in \mathbb{F}^{*}$ let $\zeta_{\lambda, \mu}\left(z^{2} x\right):=\lambda\left(z^{2}\right) \mu(x) c\left(x, z^{2}\right)$. Then $\zeta_{\lambda, \mu}$ is a well-defined splitting of the cocycle restricted to $\mathbb{E}^{* 2} \mathbb{F}^{*}$.

Conversely if $\zeta$ is any splitting of the cocycle restricted to $\mathbb{E}^{* 2} \mathbb{F}^{*}$ then $\zeta=\zeta_{\lambda, \mu}$ with $\lambda=\left.\zeta\right|_{\mathbb{E}^{* 2}}$ and $\mu=\left.\zeta\right|_{\mathbb{F}^{*}}$.

Proof. Let $\zeta$ be any splitting of the cocycle. Then $\zeta\left(z^{2} x\right)=\zeta\left(z^{2}\right) \zeta(x) c\left(x, z^{2}\right)$ by (6) and the second assertion is immediate.

Given $\lambda$ and $\mu$, choose any splitting $\zeta$. Then $\lambda$ and $\left.\zeta\right|_{\mathbb{E}^{* 2}}$ both define splittings, so $\lambda\left(z^{2}\right)=\zeta\left(z^{2}\right) \alpha\left(z^{2}\right)$ for some character $\alpha$ of $\mathbb{E}^{* 2}$. Similarly $\mu(x)=\zeta(x) \beta(x)$ for some character $\beta$ of $\mathbb{F}^{*}$. Let $\tau$ be a character of $\mathbb{E}^{*}$ extending $\alpha$ and $\beta$; this exists since, for $x \in \mathbb{E}^{* 2} \cap \mathbb{F}^{*}, \lambda(x)=\mu(x)$ implies $\alpha(x)=\beta(x)$. Then

$$
\begin{aligned}
\lambda\left(z^{2}\right) \mu(x) c\left(x, z^{2}\right) & =\zeta\left(z^{2}\right) \alpha\left(z^{2}\right) \zeta(x) \beta(x) c\left(x, z^{2}\right) \\
& =\zeta\left(z^{2} x\right) \alpha\left(z^{2}\right) \beta(x) \\
& =\zeta\left(z^{2} x\right) \tau\left(z^{2} x\right) .
\end{aligned}
$$

This shows that $\zeta_{\lambda, \mu}$ is well-defined and is a splitting of the cocycle.

Lemma 5.2. (1) Choose a character $\alpha$ of $\mathbb{E}^{*}$ satisfying

$$
\alpha(-1)=(-1,-1)_{\mathbb{F}} \gamma_{\mathbb{E}}\left(-1, \psi_{\mathbb{E}}\right)
$$

and let

$$
\lambda_{\alpha}\left(z^{2}\right)=c(z, z) \gamma_{\mathbb{E}}\left(z, \psi_{\mathbb{E}}\right) \gamma_{\mathbb{F}}\left(N z, \psi_{\mathbb{F}}\right) \alpha(z)
$$


Then $\lambda_{\alpha}$ is a well-defined splitting of the cocycle restricted to $\mathbb{E}^{* 2}$.

Furthermore every splitting of the cocycle restricted to $\mathbb{E}^{* 2}$ is equal to

$\lambda_{\alpha}$ for some $\alpha$ satisfying (13).

(2) Let $\beta$ be a character of $\mathbb{F}^{*}$ and let

$$
\mu_{\beta}(x)=\gamma_{\mathbb{F}}\left(x, \psi_{\mathbb{F}}\right) \beta(x) .
$$

Then $\mu_{\beta}$ is a splitting of the cocycle restricted to $\mathbb{F}^{*}$, and every splitting of the cocycle restricted to $\mathbb{F}^{*}$ is equal to $\mu_{\beta}$ for some $\beta$.

(3) Suppose $\alpha \in \widehat{\mathbb{E}^{*}}, \beta \in \widehat{\mathbb{F}^{*}}$ satisfy

$$
\alpha(z)=\gamma_{\mathbb{F}}\left(z^{2}, \psi_{\mathbb{F}}\right) \gamma_{\mathbb{F}}\left(N z, \psi_{\mathbb{F}}\right) \gamma_{\mathbb{E}}\left(z, \psi_{\mathbb{E}}\right) c(z, z) \beta\left(z^{2}\right) \quad\left(z^{2} \in \mathbb{F}^{*}\right) .
$$

In particular $\alpha$ satisfies (13). For $z \in \mathbb{E}^{*}, x \in \mathbb{F}^{*}$ define

$$
\begin{aligned}
\zeta_{\alpha, \beta}\left(z^{2} x\right) & :=\lambda_{\alpha}\left(z^{2}\right) \mu_{\beta}(x) c\left(x, z^{2}\right) \\
& =\gamma_{\mathbb{E}}\left(z, \psi_{\mathbb{E}}\right) \gamma_{\mathbb{F}}\left(N z, \psi_{\mathbb{F}}\right) \gamma_{\mathbb{F}}\left(x, \psi_{\mathbb{F}}\right) \alpha(z) \beta(x) c(z, z) c\left(x, z^{2}\right) .
\end{aligned}
$$

Then $\zeta$ is a well-defined splitting of the cocycle restricted to $\mathbb{E}^{* 2} \mathbb{F}^{*}$. Furthermore every splitting of the cocycle restricted to $\mathbb{E}^{* 2} \mathbb{F}^{*}$ is equal to $\zeta_{\alpha, \beta}$ for some $\alpha, \beta$ satisfying (14).

Proof. Part (1) is an extension of (8) to the case of a non-abelian group. Thus by (8) and (11),

$$
\begin{aligned}
c\left(z^{2}, w^{2}\right) & =c(z, z) c(w, w) c(z w, z w)\{z, w\} \\
& =c(z, z) c(w, w) c(z w, z w)(z, w)_{\mathbb{E}}(N z, N w)_{\mathbb{F}} .
\end{aligned}
$$

Replacing $(z, w)_{\mathbb{E}}$ by $\gamma_{\mathbb{E}}\left(z, \psi_{\mathbb{E}}\right) \gamma_{\mathbb{E}}\left(w, \psi_{\mathbb{E}}\right) \gamma_{\mathbb{E}}\left(w z, \psi_{\mathbb{E}}\right)^{-1}$, and similarly $(N z, N w)_{\mathbb{F}}$ gives

$$
c\left(z^{2}, w^{2}\right)=\tau(z) \tau(w) \tau(z w)^{-1}
$$

with $\tau(z)=c(z, z) \gamma_{\mathbb{E}}\left(z, \psi_{\mathbb{E}}\right) \gamma_{\mathbb{F}}\left(N z, \psi_{\mathbb{F}}\right)$. The same relation holds with $\tau(z)$ replaced by $\tau(z) \alpha(z)$.

We check the condition that $\lambda_{\alpha}\left(z^{2}\right):=\tau(z) \alpha(z)$ be well-defined:

$$
\begin{aligned}
& \tau(-z) \alpha(-z) \\
& =c(-z,-z) \gamma_{\mathbb{E}}\left(-z, \psi_{\mathbb{E}}\right) \gamma_{\mathbb{E}}\left(N(-z), \psi_{\mathbb{E}}\right) \alpha(-z) \\
& =c(-z,-z) \gamma_{\mathbb{E}}\left(-1, \psi_{\mathbb{E}}\right) \gamma_{\mathbb{E}}\left(z, \psi_{\mathbb{E}}\right)(-1, z)_{\mathbb{E}} \gamma_{\mathbb{F}}\left(N z, \psi_{\mathbb{F}}\right) \alpha(z) \alpha(-1) .
\end{aligned}
$$

A simple calculation using (10) gives

$$
c(-z,-z)=(-1,-1)_{\mathbb{F}}(-1, N z)_{\mathbb{F}} c(z, z)
$$

and inserting this gives

$$
\begin{aligned}
\tau(-z) \alpha(-z) & =(-1,-1)_{\mathbb{F}} \gamma_{\mathbb{E}}\left(-1, \psi_{\mathbb{E}}\right) \alpha(-1) \tau(z) \alpha(z) \\
& =\tau(z) \alpha(z) \quad \text { by }(13) .
\end{aligned}
$$


Fix $\alpha$ satisfying (13). If $\lambda$ is any splitting of the cocycle restricted to $\mathbb{E}^{* 2}$ then $\lambda=\lambda_{\alpha} \delta$ for some character $\delta$ of $\mathbb{E}^{* 2}$. Extend $\delta$ to a character $\delta^{*}$ of $\mathbb{E}^{*}$. Then $\lambda_{\alpha} \delta=\lambda_{\alpha \delta^{* 2}}$, and $\alpha \delta^{* 2}$ satisfies (13). This proves (1).

By $(10), c(\operatorname{diag}(x, x), \operatorname{diag}(y, y))=(x, y)_{\mathbb{F}}$, and (2) follows as in Section 3.

For (3) apply Lemma 5.1. Inserting $\lambda_{\alpha}, \mu_{\beta}$ in the condition of the Lemma gives (14). The final assertion follows as in the proof of (1). This completes the proof.

Let $\zeta$ be any splitting of the cocycle restricted to $\mathbb{E}^{* 2} \mathbb{F}^{*}$. Then $\zeta=\zeta_{\alpha, \beta}$ for some $\alpha, \beta$ satisfying (14). This implies:

Lemma 5.3. The map $\alpha(z):=\gamma_{\mathbb{F}}\left(z^{2}, \psi_{\mathbb{F}}\right) \gamma_{\mathbb{F}}\left(N z, \psi_{\mathbb{F}}\right) \gamma_{\mathbb{E}}\left(z, \psi_{\mathbb{E}}\right) c(z, z)$ is a character of $\left\{z \in \mathbb{E}^{*} \mid z^{2} \in \mathbb{F}^{*}\right\}$.

For completeness we also prove this directly:

$$
\begin{aligned}
\alpha(z w)= & \gamma_{\mathbb{F}}\left(z^{2} w^{2}, \psi_{\mathbb{F}}\right) \gamma_{\mathbb{F}}\left(N(z w), \psi_{\mathbb{F}}\right) \gamma_{\mathbb{E}}\left(z w, \psi_{\mathbb{E}}\right) c(z w, z w) \\
= & \gamma_{\mathbb{F}}\left(z^{2}, \psi_{\mathbb{F}}\right) \gamma_{\mathbb{F}}\left(w^{2}, \psi_{\mathbb{F}}\right)\left(z^{2}, w^{2}\right)_{\mathbb{F}} \gamma_{\mathbb{F}}\left(N z, \psi_{\mathbb{F}}\right) \gamma_{\mathbb{F}}\left(N w, \psi_{\mathbb{F}}\right)(N z, N w)_{\mathbb{F}} \\
& \gamma_{\mathbb{E}}\left(z, \psi_{\mathbb{E}}\right) \gamma_{\mathbb{E}}\left(w, \psi_{\mathbb{E}}\right)(z, w)_{\mathbb{E}} c(z w, z w) \\
= & \alpha(z) \alpha(w) c(z, z) c(w, w) c\left(z^{2}, w^{2}\right)\{z, w\} c(z w, z w) \quad \text { by }(11) \\
= & \alpha(z) \alpha(w) \quad \text { by }(8) .
\end{aligned}
$$

Remark 5.4. Given $\alpha \in \widehat{\mathbb{E}^{*}}$ there exists $\beta \in \widehat{\mathbb{F}^{*}}$ satisfying (14) if and only if (13) holds.

This follows by an argument as in the proof of Lemma 5.2 (1): Define $\beta\left(z^{2}\right)=\alpha(z) \gamma_{\mathbb{F}}\left(z^{2}, \psi_{\mathbb{F}}\right)^{-1} \gamma_{\mathbb{F}}\left(N z, \psi_{\mathbb{F}}\right)^{-1} \gamma_{\mathbb{E}}\left(z, \psi_{\mathbb{E}}\right)^{-1} c(z, z)$; this is welldefined if (13) holds, and extends to a character of $\mathbb{F}^{*}$.

Suppose $z \in \mathbb{E}^{1}$. By Hilbert's Theorem $90, z=w / \bar{w}$ for some $w \in \mathbb{E}^{*}$. Then $z=w^{2} / N(w) \in \mathbb{E}^{* 2} \mathbb{F}^{*}$, so $\zeta$ restricts to a splitting of the extension of $\mathbb{E}^{1}$. We now make explicit choices such that $\zeta$ is a $\mathbb{T}$ or $\mu_{n}$ splitting as in Theorem 1.

Lemma 5.5. We may choose $\psi_{\mathbb{E}}$ so that

$$
\gamma_{\mathbb{E}}\left(x, \psi_{\mathbb{E}}\right)=1 \quad\left(x \in \mathbb{F}^{*}\right) .
$$

Proof. Since $\gamma_{\mathbb{E}}\left(\cdot, \psi_{\mathbb{E}}\right)$ is a quadratic character of $\mathbb{F}^{*}, \gamma_{\mathbb{E}}\left(x, \psi_{\mathbb{E}}\right)=(x, y)_{\mathbb{F}}$ for some $y \in \mathbb{F}^{*}$. Suppose $\mathbb{E}=\mathbb{F}(\sqrt{\Delta})$. Then $\gamma_{\mathbb{E}}\left(\Delta, \psi_{\mathbb{E}}\right)=1$ since $\Delta$ is a square in $\mathbb{E}^{*}$. Therefore $(\Delta, y)_{\mathbb{F}}=1$, so $y=N w$ for some $w \in \mathbb{E}^{*}$. Replacing $\psi_{\mathbb{E}}$ by $w \psi_{\mathbb{E}}$ gives (cf. [10], Appendix)

$$
\begin{aligned}
\gamma_{\mathbb{E}}\left(x, w \psi_{\mathbb{E}}\right) & =(x, w)_{\mathbb{E}} \gamma_{\mathbb{E}}\left(x, \psi_{\mathbb{E}}\right) \\
& =(x, N w)_{\mathbb{F}}(x, y)_{\mathbb{F}}=1 .
\end{aligned}
$$

This completes the proof. 
Lemma 5.6. Fix $\psi_{\mathbb{E}}$ satisfying Lemma 5.5. Choose $\alpha, \beta$ satisfying (14) and let $\zeta=\zeta_{\alpha, \beta}$. Then

$$
\zeta(z)^{2}=\alpha(z) \quad\left(z \in \mathbb{E}^{1}\right) .
$$

Proof. Writing $z=w / \bar{w}=w^{2} / N(w)$ and applying the definition (15) gives

$$
\zeta(w / \bar{w})=(-1, N w)_{\mathbb{F}} \gamma_{\mathbb{E}}\left(w, \psi_{\mathbb{E}}\right) \alpha(w) \beta\left(N w^{-1}\right) c(w, w) c\left(N w^{-1}, w^{2}\right)
$$

and

$$
\begin{aligned}
\zeta(w / \bar{w})^{2} & =(-1, w)_{\mathbb{E}} \alpha\left(w^{2}\right) \beta\left(N w^{-2}\right) \\
& =(-1, N w)_{\mathbb{F}} \alpha\left(w^{2}\right) \alpha\left(N w^{-2}\right)(-1, N w)_{\mathbb{F}} \gamma_{\mathbb{E}}\left(N w^{-1}, \psi_{\mathbb{E}}\right) \quad \text { by }(14) \\
& =\alpha\left(w / \bar{w} \gamma_{\mathbb{E}}\left(N w, \psi_{\mathbb{E}}\right)\right. \\
& =\alpha(w / \bar{w}) \quad \text { by }(17) .
\end{aligned}
$$

We see that $\zeta_{\alpha, \beta}$ is a $\mu_{2 n}$ splitting if and only if $\alpha(z)^{n}=1$ for all $z \in \mathbb{E}^{1}$. We now complete the proof of Theorem 1 .

Proof of Theorem 1. By (16) and (17) we have

$$
\alpha(-1)=(-1,-1)_{\mathbb{F}} .
$$

Therefore we may choose $\alpha=1$ if $(-1,-1)_{\mathbb{F}}=1$. Assume $(-1,-1)_{\mathbb{F}}=-1$. If $\mathbb{F}=\mathbb{R}$ then $\alpha(z)=z^{n}$ for $n$ odd as in Section 4 , so assume $\mathbb{F}$ is nonarchimedean. If $-1 \notin \mathbb{E}^{* 2}$ we may choose $\alpha^{2}=1$. If $-1 \in \mathbb{E}^{* 2}$ then $-1 \notin \mathbb{E}^{* 4}$ (cf. $\S 4$ ) and we may choose $\alpha^{4}=1$.

We make some explicit choices and summarize the preceding discussion.

If $(-1,-1)_{\mathbb{F}}=-1$ and $-1 \notin \mathbb{E}^{* 2}$ choose $z_{1} \in \mathbb{E}^{*}$ with $\left(z_{1},-1\right)_{\mathbb{E}}=-1$.

If $(-1,-1)_{\mathbb{F}}=-1$ and $-1 \in \mathbb{E}^{* 2}$, i.e., $\mathbb{E}=\mathbb{F}(\sqrt{-1})$, then the norm residue symbol $(w, z)_{\mathbb{E}, 4}$ is defined. In particular the map $z \rightarrow(w, z)_{E, 4}$ is a character of $\mathbb{E}^{*}$ of order 4 . Choose $z_{2} \in \mathbb{E}^{*}$ satisfying $\left(z_{2},-1\right)_{\mathbb{E}, 4}=-1$.

Theorem 5.7. Choose a non-trivial character $\psi_{\mathbb{E}}$ of $\mathbb{E}$ such that $\gamma_{\mathbb{E}}\left(x, \psi_{\mathbb{E}}\right)=$ 1 for all $x \in \mathbb{F}^{*}$ (Lemma 5.5). For $z \in \mathbb{E}^{*}$ let

$$
\alpha(z):= \begin{cases}z & \mathbb{F}=\mathbb{R} \\ 1 & (-1,-1)_{\mathbb{F}}=1 \\ \left(z_{1}, z\right)_{\mathbb{E}} & (-1,-1)_{\mathbb{F}}=-1,-1 \notin \mathbb{E}^{* 2} \\ \left(z_{2}, z\right)_{\mathbb{E}, 4} & (-1,-1)_{\mathbb{F}}=-1,-1 \in \mathbb{E}^{* 2} .\end{cases}
$$

Then $\alpha$ is a character of $\mathbb{E}^{*}$ of order $\infty, 1,2$ or 4 respectively, satisfying (13). Choose a character $\beta$ of $\mathbb{F}^{*}$ satisfying

$$
\beta\left(z^{2}\right)=\gamma_{\mathbb{F}}\left(z^{2}, \psi_{F}\right)^{-1} \gamma_{\mathbb{F}}\left(N z, \psi_{\mathbb{F}}\right)^{-1} \gamma_{\mathbb{E}}\left(z, \psi_{\mathbb{E}}\right)^{-1} c(z, z) \alpha(z) \quad\left(z^{2} \in \mathbb{F}^{*}\right)
$$


(cf. Remark 5.4). In particular

$$
\beta\left(x^{2}\right)=(-1, x)_{\mathbb{F}} \alpha(x) \quad\left(x \in \mathbb{F}^{*}\right) .
$$

Let

$$
\zeta(w / \bar{w})=\gamma_{\mathbb{E}}\left(w, \psi_{\mathbb{E}}\right) \alpha(w) \beta\left(N w^{-1}\right) c(w, w) c\left(N w^{-1}, w^{2}\right) .
$$

Then $\zeta$ is a splitting of the cocycle restricted to $\mathbb{E}^{1}$. Furthermore for $z \in \mathbb{E}^{1}$ $\zeta(x)^{2}=\alpha(z)$, and for $\mathbb{F}$ non-archimedean this gives $\zeta(z)^{n}=\alpha(z)^{n / 2}=1$ with

$$
n= \begin{cases}2 & (-1,-1)_{\mathbb{F}}=1 \\ 4 & (-1,-1)_{\mathbb{F}}=-1,-1 \notin \mathbb{E}^{* 2} \\ 8 & (-1,-1)_{\mathbb{F}}=-1,-1 \in \mathbb{E}^{* 2} .\end{cases}
$$

Remark 5.8. With $\alpha, \beta$ as in the Theorem,

$$
\zeta(w / \bar{w})=\zeta_{\alpha, \beta}(w / \bar{w})(-1, N w)_{\mathbb{F}} .
$$

We have dropped the term $(-1, N w)$, which is allowed since $w / \bar{w} \rightarrow$ $(-1, N w)$ is a quadratic character of $\mathbb{E}^{1}$.

Henceforth write $\mathbb{E}=\mathbb{F}(\delta)$ with $\Delta:=\delta^{2} \in \mathbb{F}$.

Remark 5.9. Condition (20) is equivalent to

$$
\begin{aligned}
\beta\left(x^{2}\right) & =(-1, x)_{\mathbb{F}} \alpha(x) \quad\left(x \in \mathbb{F}^{*}\right) \\
\beta(\Delta) & =\gamma_{\mathbb{F}}\left(-1, \psi_{\mathbb{F}}\right)^{-1} \gamma_{\mathbb{E}}\left(\delta, \psi_{\mathbb{E}}\right)^{-1} c(\delta, \delta) \alpha(\delta) .
\end{aligned}
$$

The splitting has a simple formula on $T^{2}$ :

$$
\zeta\left(z^{2}\right)=c(z, z) \alpha(z) \quad\left(z \in \mathbb{E}^{1}\right),
$$

which is independent of $\beta, \psi_{\mathbb{F}}$ and $\psi_{\mathbb{E}}$. Note that any two $\mu_{2}$ splittings of $T$ have the same restriction to $T^{2}$ since they differ by a quadratic character.

The map $w / \bar{w} \rightarrow N w$ induces an isomorphism $T / T^{2} \simeq N \mathbb{E}^{*} / \mathbb{F}^{* 2}$. Choose representatives $a_{1}, \ldots, a_{n}$ of generators of $N \mathbb{E}^{*} / \mathbb{F}^{* 2}$, with corresponding elements $z_{1}, \ldots, z_{n} \in T$. Given $\alpha$ there are two choices of each $\beta\left(a_{i}\right)$ differing by sign, and these signs may may be chosen arbitrarily. The following result follows easily.

Corollary 5.10. Choose representatives $z_{1} \ldots, z_{m}$ of generators of $T / T^{2} \simeq$ $N \mathbb{E}^{*} / \mathbb{F}^{* 2}$. Choose $\alpha$ as in Theorem 5.7. Define

$$
\zeta\left(z^{2}\right)=c(z, z) \alpha(z) \quad\left(z \in \mathbb{E}^{1}\right)
$$

and for $1 \leq i \leq m$ let $\zeta\left(z_{i}\right)$ be either square root of $\alpha\left(z_{i}\right)$. Then $\zeta$ extends uniquely to a splitting of the cocycle as in Theorem 5.7. 
In the non-archimedean case by ([4], Lemma 0.3.2) $\left|T / T^{2}\right|=2 /|2|_{\mathbb{F}}$, which equals 2 if the residual characteristic of $\mathbb{F}$ is odd.

We conclude with a few remarks about the definition of $\zeta$.

From the definition we have for $w \in \mathbb{E}^{*}$ :

$$
c(w, w)=\left(-N w, x(w) x\left(w^{2}\right)\right)_{\mathbb{F}}
$$

and

$$
c\left(N w^{-1}, w^{2}\right)=\left(N w, x\left(w^{2}\right)\right)_{\mathbb{F}} .
$$

Note that for $\lambda \in \mathbb{F}^{*}, w \in \mathbb{E}^{*}$ we have

$$
c(\lambda, w)=(\lambda, x(w))_{\mathbb{F}} .
$$

Fix $u \in \mathbb{E}^{*}$ with $\operatorname{trace}(u)=0$, and define $\operatorname{Tr}_{u}: \mathbb{E}^{*} \rightarrow \mathbb{F}^{*}$ by

$$
\operatorname{Tr}_{u}(z)= \begin{cases}\operatorname{trace}(z) & \operatorname{trace}(z) \neq 0 \\ u z & \operatorname{trace}(z)=0\end{cases}
$$

Up to conjugation by $S L(2, \mathbb{F})$ we may assume $\iota(x+y \delta)=\left(\begin{array}{cc}x & y \Delta / a \\ y a & x\end{array}\right)$ for some $a \in \mathbb{F}^{*}$. If $w=x+y \delta$ with $x y \neq 0$ we have $x(w) x\left(w^{2}\right)=2 x y^{2} a^{2}$. Considering the cases with $x y=0$ separately gives

$$
c(w, w)=\left(-N w, \operatorname{Tr}_{a \delta}(w)\right)_{\mathbb{F}} \quad\left(z \in \mathbb{E}^{*}\right)
$$

and

$$
\zeta\left(z^{2}\right)=\left(-1, \operatorname{Tr}_{a \delta}(z)\right)_{\mathbb{F}} \alpha(z) \quad\left(z \in \mathbb{E}^{1}\right) .
$$

For example if $p$ is odd and $\mathbb{E} \neq \mathbb{F}(\sqrt{-1})$ then $m=1$ (cf. Corollary 5.10), we may take $z_{1}=-1, \alpha=1$ and $\zeta(-1)=1$ which gives

$$
\zeta\left(\epsilon z^{2}\right)=c(z, z) c\left(\epsilon, z^{2}\right) \quad\left(\epsilon= \pm 1, z \in \mathbb{E}^{1}\right) .
$$

For example let $\mathbb{F}=\mathbb{R}$ and define $\iota(x+i y)=\left(\begin{array}{cc}x & y \\ -y & x\end{array}\right)$. We take $\alpha(z)=z$ and $\beta(x)= \pm \sqrt{|x|}$. Then for $z \in \mathbb{E}^{1}$,

$$
\zeta\left(z^{2}\right)=\left(-1, \operatorname{Tr}_{-i}(z)\right)_{\mathbb{R}} z=\operatorname{sgn}\left(\operatorname{Tr}_{-i}(z)\right) z
$$

(independent of $\beta$ ). Note that $a=-1$ and $\operatorname{Tr}_{-i}(i y)=y\left(y \in \mathbb{R}^{*}\right.$ ).

\section{References}

[1] K.S. Brown, Cohomology of Groups, Number 87 in Graduate Texts in Mathematics, Springer-Verlag, Berlin-Heidelberg-New York, 1982, MR 83k:20002, Zbl 584.20036.

[2] L. Calabi, Sur les extensions des groupes topologiques, Ann. Mat. Pura Appl., 32(4) (1951), 295-370, MR 14,245d, Zbl 054.01302.

[3] Y. Flicker, Automorphic forms on covering groups of GL(2), Invent. Math., 57 (1980), 119-182, MR 81m:10057, Zbl 431.10014. 
[4] D.A. Kazhdan and S.J. Patterson, Metaplectic forms, Inst. Hautes Études Sci. Publ. Math., 59 (1984), 35-142, MR 87h:22024, Zbl 559.10026.

[5] _ Towards a generalized Shimura correspondence, Adv. in Math., 60(2) (1986), 161-234, MR 87m:22050, Zbl 616.10023.

[6] T. Kubota, Automorphic forms and the reciprocity law in a number field, preprint, Kyoto University, 1969.

[7] S. Kudla, Splitting metaplectic covers of dual reductive pairs, Israel J. Math., 87 (1994), 361-401, MR 95h:22019, Zbl 840.22029.

[8] C. Moore, Extensions and low-dimensional cohomology theory of locally compact groups. II, Trans. Amer. Math. Soc., 113 (1964), 64-86, MR 30 \#2106, Zbl 131.26902.

[9] - Group extensions of p-adic and adelic linear groups, Inst. Hautes Études Sci. Publ. Math., 35 (1968), 157-222, MR 39 \#5575, Zbl 159.03203.

[10] R. Ranga Rao, On some explicit formulas in the theory of the Weil representation, Pacific J. Math., 157 (1993), 335-371, MR 94a:22037, Zbl 794.58017.

[11] J. Rogawski and S. Gelbart, L-functions and Fourier-Jacobi coefficients for the unitary group U(3), Invent. Math., 105 (1991), 445-472, MR 93b:11059, Zbl 742.11030.

Received April 29, 1999.

Department of Mathematics

UNIVERSITY OF MARYLAND

College Park, MD 20742

E-mail address: jda@math.umd.edu 